\title{
Multicriteria scheduling problem: a hybrid ant colony algorithm integrating the decision-maker's preferences
}

\author{
Mohamed Anis Allouche*, Tahar Jouili, Mohamed Ali Omri \\ College of Business Administration, Northern Border University, Arar, Saudi Arabia
}

\section{A R T I C L E IN F O}

\section{Article history:}

Received 18 May 2017

Received in revised form

8 August 2017

Accepted 8 August 2017

\section{Keywords:}

Multicriteria scheduling problem

Ant colony algorithm

Variable neighborhood search

Satisfaction functions

Decision-maker's preferences

\section{Introduction}

As defined by T'kindt and Billaut (2002), a scheduling problem consists of finding the sequence of a set of jobs (tasks) to be processed on different machines, so that technological constraints are satisfied and one or several performance criteria are optimized. Generally, the objectives of scheduling problem are conflicting in nature. Indeed, it is generally impossible to find a sequence in which all conflicting scheduling criteria are optimized simultaneously. Hence, the decision maker needs to make some trade-offs between the scheduling criteria in order to obtain the most satisfactory sequence. Several researchers have studied the problem of multi-criteria scheduling flow shop type. Gangadharan and Rajendran (1994) have considered the minimization of the makespan and the total completion time criteria. They applied a simulated annealing technique to develop their own heuristics. Rajendran and Ziegler (2004) proposed two ant colonies algorithms to solve a scheduling problem in order to minimize simultaneously the makespan and total completion time. Lin et al. (2008) have introduced new features in an ant colony algorithm inspired by the behavior of ants to develop a new algorithm and produce better solutions. Loukil et al.

\footnotetext{
* Corresponding Author.

Email Address: medanis.allouche@yahoo.fr (M. A. Allouche) https://doi.org/10.21833/ijaas.2017.09.023

2313-626X/C) 2017 The Authors. Published by IASE.

This is an open access article under the CC BY-NC-ND license

(http://creativecommons.org/licenses/by-nc-nd/4.0/)
}

(2005), Varadharajan and Rajendran (2005) have adapted a multi-objective simulated annealing (MOSA) method to solve a multicriteria scheduling problem.

There are several other studies that have been developed in the area of multi-criteria scheduling problem such as: Tavakkoli-Moghaddam et al. (2007), Eren (2007), Javadi et al. (2008), Chang et al. (2008), Ruiz and Allahverdi (2009) and Qian et al. (2009). T'kindt and Billaut (2002) presented a quite complete literature review regarding multi-criteria scheduling theory. These entire works do not take into account, explicitly, the decision maker's preferences in the decisional process. Nevertheless, small numbers of proposed approaches cited in the literature, consider the decision maker's preferences in the multicriteria scheduling problems. Gagné et al. (2004) treated a problem of industrial multiobjective scheduling a single machine with dependent setup times. They proposed a generic procedure to search a compromise solutions based on ant colonies algorithm which takes into account the preferences set by the decision maker. This procedure based on the following three steps: a) determining the ideal point, b) determining the compromise solution and c) presenting effective solutions to the decision maker. For multi-objective scheduling problems, Gagné et al. (2005) have used metaheuristics, based on hybrid Tabu Search/Variable Neighborhood Search (Tabu-VNS), to finding compromise solutions. In the same context, Allouche et al. (2009) have presented an exact method to solve a multi-criteria permutation 
flowshop problem. This approach based on compromise programming model and the concept of satisfaction functions and that, by incorporating explicitly the preferences of the decision maker. The satisfaction functions measure the intensity of preference regarding the deviations between the achievement and the aspiration levels of the following criteria: makespan, total flow time and total tardiness. Allouche (2010) has proposed a Tabu Search metaheuristic to solve permutation flow shop scheduling problem where several criteria are to be considered, i.e., the makespan, total flowtime and total tardiness of jobs. The Compromise Programming model and the concept of satisfaction functions are used to integrate explicitly the manager's preferences. In this paper, we propose a new metaheuristic approach based on ant colony optimization and variable neighborhood search to solve the permutation flowshop scheduling problem by considering the same criteria used in Allouche (2010). The proposed metaheuristic approach incorporates explicitly the manager's preferences by using the concept of satisfaction functions. It will be tested through a computational experiment and compared to results obtained by Allouche (2010).

This paper is organized as follows. Components and steps of the proposed metaheuristic are given in section 2 and 3 respectively. In section 4, Numerical experiments on several multicriteria scheduling problems are presented and computational results and discussions are presented in section 5 . Section 6 concludes the paper with making the focus to the advantages and limits of our work.

\section{Metaheuristic components}

In this section, we present the different components of the proposed metaheuristic. In fact, it is based on the three main elements: the compromise programming model, the satisfaction functions concept and the hybrid ant colony algorithm.

\subsection{The compromise programming model}

The Compromise Programming model (CP) was developed first by Zeleny (1973). It is based on minimizing the distance between the achievement level of the objective $q\left(f_{q}(x)\right.$ and the ideal value $\left(g_{q}^{*}\right)$ associated with this objective. The value can be obtained according to the objective of optimization, and the mathematical model form of the CP model is as follows:

\begin{tabular}{c||c} 
Aim to maximise & Aim to minimise \\
\hline $\operatorname{Min} \sum_{q=1}^{Q} W_{q}^{-} \delta_{q}^{-}$ & $\operatorname{Min} \sum_{q=1}^{Q} W_{q}^{+} \delta_{q}^{+}$ \\
Subject to: & Subject to: \\
$f_{q}(x)+\delta_{q}^{-}=g_{q}^{*}$ & $f_{q}(x)-\delta_{q}^{+}=g_{q}^{*}$ \\
$(\forall q \in Q)$ & $(\forall q \in Q)$ \\
$x \in F$ & $x \in F$ \\
$\delta_{q}^{-} \geq 0(\forall q \in Q)$ & $\delta_{q}^{+} \geq 0(\forall q \in Q)$.
\end{tabular}

where:
$W_{q}^{+}$and $W_{q}^{-}$are, respectively, the weights of positive and negative deviations of the objective $q$. F: the set of feasible solutions

\subsection{The concept of satisfaction functions}

The concept of satisfaction functions was introduced in the "Goal Programming model" by Martel and Aouni (1990). These functions enable the integration of the decision maker's preferences, and this, in connection with the deviations between the levels of achievement and aspiration goals (these funds are set by the decision maker). These functions have the following general form (Fig. 1):

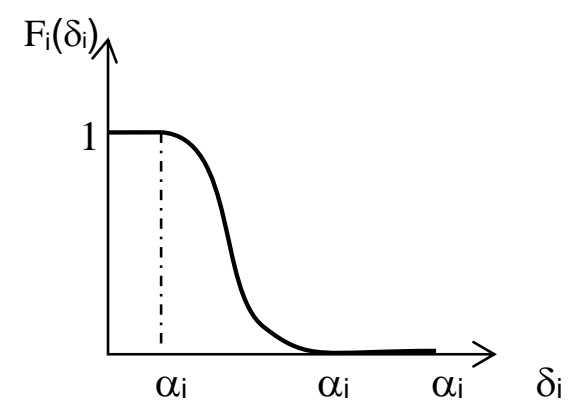

Fig. 1: General form of a satisfaction function

where:

$F_{q}\left(\delta_{q}\right)$ : Value of the satisfaction function associated with deviations $\delta_{q}$,

$\alpha_{q d}$ : Indifference threshold,

$\alpha_{q 0}$ : Nil satisfaction threshold,

$\alpha_{q v}$ : Veto threshold.

$\delta_{q} \in\left[0, \alpha_{q d}\right]:$ Within this interval, the decision maker's satisfaction level reaches its maximum value of 1 .

$\delta_{q} \in\left[\alpha_{q d}, \alpha_{q o}\right]:$ Within this interval, the decision maker's satisfaction function is monotonously decreasing.

$\delta_{q} \in\left[\alpha_{q o}, \alpha_{q v}\right]:$ Within this interval, the decision maker's satisfaction function is equal to zero. When $\delta_{q}$ exceeds the veto threshold, the solution will be rejected by the decision maker.

Note that many other shapes of satisfaction functions can be found in Martel and Aouni (1990).

\subsection{The hybrid ant colony algorithm}

The proposed approach is a hybridation of two metaheuristics. The first one is based on ant colony algorithm and the second one is based on the variable neighborhood search (VNS). Note that the Ant colony Optimization (ACO) heuristic was first introduced by Dorigo et al. (1996). It is inspired by the real-life behavior of ants. Several kinds of ant colony algorithm are cited in the literature such as the "Ant System (AS)", proposed by Dorigo et al. (1996), the "Ant Colony System (ACS)" proposed by Gambardella and Dorigo (1996), the "Ant-Q" proposed by Gambardella and Dorigo (1995). In this 
paper, we used ACS algorithm which is detailed in Fig. 2.

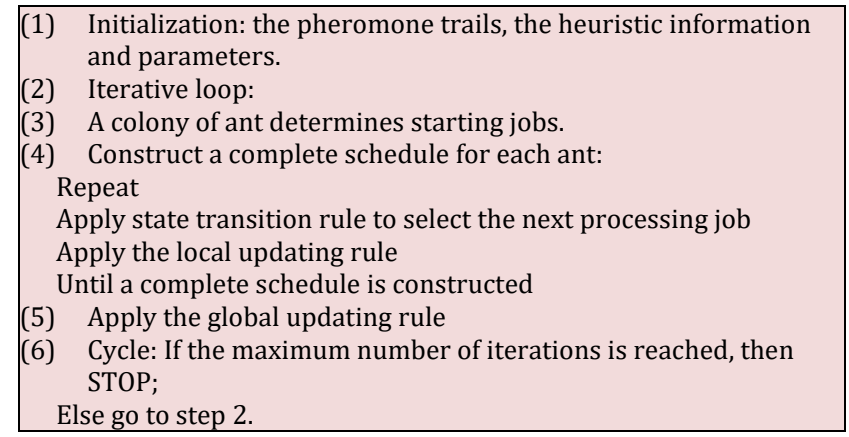

Fig. 2: The ACS algorithm

On the other hand, variable neighborhood search (VNS) is one of the recently proposed metaheuristics in the field of combinatorial optimization. It was first introduced by Hansen and Mladenović (1997). It is based on the systematic change of the neighborhood within a possibly randomized local search algorithm. Contrary to other metaheuristics based on local search methods, VNS does not follow a trajectory but explores increasingly distant neighborhoods of a current solution, and jumps from it to a new one if and only if an improvement has been made.

A major advantage of VNS is that it avoids any blockage in a local optimum. Using VNS needs to set some parameters such as: a) the adopted neighborhood structures and b) the order of implementation of these structures. The VNS algorithm is represented in Fig. 3.

1. Initialization: find an initial solution $\mathrm{x}$.

2. Repeat the following steps:

a) Randomly generate a solution $x^{\prime} \in V(x)$;

b) Apply to $x^{\prime}$ procedure k local search until a maximum number of iterations

To a local optimum $x^{\prime \prime}$;

If $\mathrm{x}^{\prime}$ is better than $\mathrm{x}^{\prime \prime}$ then $\mathrm{k}=1$ otherwise $\mathrm{k}=\mathrm{k}+1$.

Fig. 3: The VNS algorithm

The structure of the proposed algorithm which is a hybridation of ant colony algorithm and variable neighborhood search is presented in Fig. 4.

[1] Initialization: the pheromone trails, the heuristic

information and parameters

[2] Iterative loop:

[3] A colony of ant determines starting jobs.

[4] Construct a complete schedule for each ant:

Repeat

Apply state transition rule to select the next processing job

Apply the local updating rule

Until a complete schedule is constructed

[5] Apply VNS

[6] Apply the global updating rule

[7] Cycle: If the maximum number of iterations is reached, then STOP;

Else go to step 2

Fig. 4: ACS-VNS algorithm

\section{Metaheuristic steps}

In this section, we present the different steps of the proposed metaheuristic approach. The first step determines the lower bounds of each criterion (ideal points) and the second step consists of determining the best compromise solutions (sequence).

\subsection{Determination of ideal points}

In this step, we optimize each criterion separately by using the hybrid ant colonies. The obtained solutions represent the ideal points $\mathrm{g}_{\mathrm{q}}{ }^{*}$.

The basic concepts of the proposed metaheuristic are presented as follows:

- The pheromone information: is presented by a matrix $\mathrm{P}(n \times n)$ where $\mathrm{n}$ is the number of tasks. It is initialized at $\tau_{0}$, a real value fixed by the users and will be modified by ants during the execution process. In this paper, $\tau_{0}$ is fixed at 0.1 . Note that $\tau_{i j}$ is considered as the intensity of the pheromone trail for all tasks pairs $(i, j)$ where $i$ is the scheduled task and $j$ the new task to be scheduled which having the maximum value of $\tau_{i j}$.

- The Heuristic information $\left(\eta_{i j}\right)$ : the choice of the next task (job) to be scheduled is influenced by heuristic information (visibility) related to the characteristics of tasks such as operating (processing) time. This information can be static or dynamic. Note that in our case, it was considered as static. We initialize the elements of matrix $\mathrm{H}$ with different values which give priority to tasks having the shortest processing time. The value of an item $\eta_{\mathrm{ij}}$ is calculated as follows:

$$
\eta_{i j}=\frac{1}{1+\sum_{r=1}^{m} p_{j r}}
$$

where:

$p_{j r}$ : is the operating time of the task $j$ on the machine $r$.

We note that the heuristic information represents, for an ant positioned on task $j$, the quality of other unscheduled tasks. Any tasks having the maximum value of $\eta_{i j}$ will be qualified as a best quality.

- The state transition rule: The choice of the next task scheduling is done by applying the state transition rule that defines how an ant $k$ in the position $i$ choose the position $j$ at time $t$. The algorithm generates a random number $q$ belonging to $[0,1]$. This number will be compared to $q_{0}$. Note that $q_{0}$ is a parameter that determines the relative importance of the exploitation of existing information (intensification) and the exploration of new solutions (diversification).

$$
\text { If }\left(q \leq q_{0}\right) \text { Then } j=\operatorname{argmax}\left\{[\tau(i, u)]^{\alpha}[\eta(i, u)]^{\beta}\right\}
$$
$u \in S_{k}(i)$, otherwise $j=J$; $J$ is chosen according to probability:

$P_{i j}^{k}(t)=\frac{[\tau(i, j)]^{\alpha}[\eta(i, j)]^{\beta}}{\sum_{u \in S_{k(i)}}[\tau(i, u)]^{\alpha}[\eta(i, u)]^{\beta}}$ 
$j \in S_{k}(i)$

where:

$S_{k}(i)$ : set of feasible tasks that an ant $k$ found in the task $i$ can select. The ant chooses the task $j$ that has the greatest value of $\mathrm{P}_{k}$.

$\left(\tau_{\mathrm{ij}}\right)$ : represents the intensity of the pheromone trail on the $\operatorname{arc}(\mathrm{i}, \mathrm{j})$.

$\alpha$ and $\beta$ parameters used to vary the relative importance of the trail intensity and the visibility.

We note that, at time $t$, from an existing partial jobs sequence, each ant $k$ chooses the next job to add using the probabilistic rule $P_{i j}^{k}(t)$ based on visibility $\left(\eta_{i j}\right)$ and the intensity of the pheromone rule $\left(\tau_{i j}\right)$.

- The local updating rule: when an ant moves from task $i$ to task $j$, it updates the value of pheromone information $\tau_{\text {ij }}$ (updated step by step). This update is done in order to reduce the value of $\tau_{i j}$ and to make the visited task less attractive for further research to other components (diversification), until a complete scheduling is built. The rule of the local update is given as follows:

$\tau(i, j)=(1-\rho l) \cdot \tau(i, j)+\rho l . \tau_{0}$

where:

$\rho l$ : a parameter that determines the amount of the reduction of the pheromone level

- The VNS method: In this step, the best-obtained solution (schedule) provided by ant colony algorithm will be considered as the initial solution. The VNS method looks for the best solution using the neighborhood exploration. In fact, three neighborhood procedures (structures) are used in the following order:

1. Insertion procedure: insert the obtained task in position $a$ to a position $b$.

2. Random permutation procedure: swapping two tasks in position $a$ and $b$.

3. Two adjacent-tasks permutation procedure: swapping two adjacent tasks in position $a$ and $a+1$.

- The global updating rule: The purpose of this update is to increase the rate of pheromone between tasks belonging to the best sequence obtained in the previous step until this iteration (intensification). The rule of the global update is given as follows:

$$
\begin{aligned}
& \tau(i, j)=(1-\rho g) \cdot \tau(i, j)+\rho g \cdot \Delta \tau(i, j) \\
& \Delta \tau(i, j)=\left\{\begin{array}{lr}
\left(L_{b}\right)^{-1} \text { if }(i, j) \in \text { best sequence } \\
0
\end{array}\right.
\end{aligned}
$$

where:

$L_{b}$ : the value of the objective function.

$\rho g$ : the global evaporation rate of pheromone, $0<\rho g<1$

\subsection{Determination of the best compromise solution}

The proposed approach takes into account the decision maker's preferences for each criterion. These preferences are expressed using the satisfaction functions. The retained satisfaction function is presented in Fig. 5.

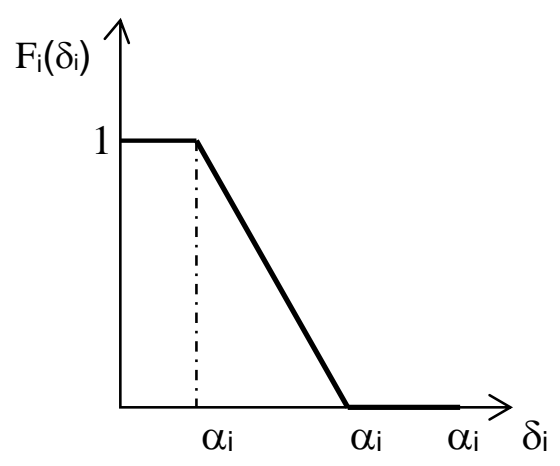

Fig. 5: Retained satisfaction function

The analytical form of this function is:

$\mathrm{F}_{1}\left(\delta_{1}\right)=\left\{\begin{array}{cc}1 & \text { if } 0 \leq \delta_{1} \leq \alpha_{q d} \\ \frac{\alpha_{q o}-\delta_{1}}{\alpha_{q o}-\alpha_{q d}} & \text { if } \alpha_{q d} \leq \delta_{1} \leq \alpha_{q o} \\ 0 & \text { if } \delta_{1}>\alpha_{q o}\end{array}\right.$

For each criterion, these thresholds, $\alpha_{q d}, \alpha_{q o}$ and $\alpha_{q v}$ are fixed by the decision maker. The retained satisfaction function will be applied to the three criteria that we are considering in our computational experiment. In addition to these concepts, the set of Pareto optimal solutions is used. It contains all nondominated sequences. In fact, the principle of dominance concerns only the value of the optimized criteria and not the value of the objective function.

\section{Numerical experiments}

The proposed metaheuristic was tested through a set of benchmark problems presented by Taillard (1993) and the obtained results are compared with those of Allouche (2010). These problems have different sizes such as 20 tasks (5-10-20 machines), 50 tasks (5-10 machines) and 100 tasks (5 machines). For each size of problem, we have tested 10 different instances. Taillard's benchmarks were served only for the makespan criterion. A series of numerical experiments is done in two steps. The first one determines the ideal point of each criterion. As to the second step, it incorporates explicitly the decision maker's preferences to determine the best compromise solution. After several experiments, the value of the following parameters is fixed at; $\alpha=\beta=2$, $\mathrm{q}_{0}=0.9, \rho g=\rho l=0.2, \mathrm{R}=0.6, \mathrm{~T}=0.4$ Note that all the computations are performed using a Pentium Centrino Duo machine, 1,73- GHz and 1-G- RAM. The proposed approach is coded in $\mathrm{C}$ language. 


\section{Computational results}

As we mentioned above, the first step consists of optimizing each criterion separately in order to obtain their best values (ideal points). Table 1 summarizes the best obtained values of the makespan $\left(\mathrm{C}^{*}\right.$ max $)$, Total flowtime $\left(\sum \mathrm{C}_{\mathrm{i}}^{*}\right)$ and Total tardiness $\left(\sum \mathrm{T}_{\mathrm{i}}^{*}\right)$ of a permutation flowshop scheduling problem characterized by 20 tasks and 10 machines ( $10 \mathrm{PF} / 20 /$ Criterion).

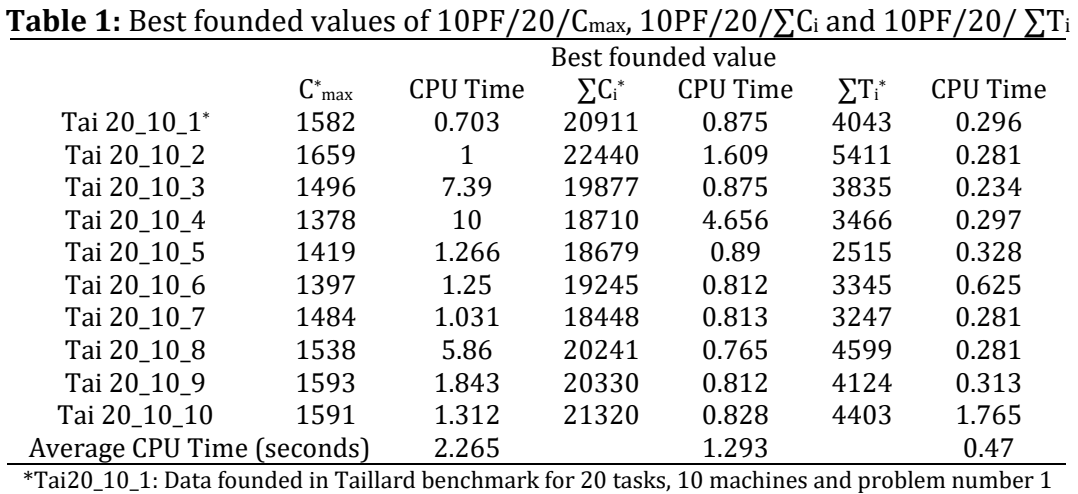

Regarding the total tardiness criterion, we considered the Daniels and chambers (1990) technique to generate due date of tasks (jobs) of different problems. The due date $\left(d_{j}\right)$ is randomly generated within the following interval:

$d_{j} \in\left[A B P\left(1-T-\frac{R}{2}\right), A B P\left(1-T+\frac{R}{2}\right)\right]$ $\mathrm{ABP}=(n+m-1) \bar{P}$

and

$\bar{P}=\frac{\sum_{i=1}^{m} \sum_{j=1}^{n} P_{i j}}{n * m}$

where:

ABP: Average Busy Period that serves as an approximation of the achievement time of the task in the sequence.

$\mathrm{T}$ : delay factor or average percentage of overdue tasks, $\mathrm{T} \in(0.4,0.6,0.8)$,

$R$ : control factor of the extent of due dates, $R \in(0.2$, $0.6,1)$,

$\bar{P}$ : Mean processing time, $n$ : number of tasks,

$m$ : number of machines.

It should be noted that the due date for each task is computed by taking $\mathrm{T}$ equal to 0.4 and $\mathrm{R}$ equal to 0.6 as done by Allouche (2010). The best-founded value of makespan, total flowtime and total tardiness illustrated in Table 1, are the same as obtained by Allouche (2010). Therefore, the ACS-VNS needs less computational time to display solutions than TS method. As mentioned in Table 1, for 20 tasks, 10 machines scheduling problem, the average computational time is 2.265 seconds, 1.293 seconds and 0.47 seconds for the makespan, total flowtime and total tardiness respectively.

The obtained results are compared with those given by Allouche (2010) only for the total flowtime and the total tardiness criteria. Note that, in their approach, Allouche (2010) were used a Tabu search method. Table 2 resumes the obtained results for scheduling problems characterized by 20 tasks and 10 machines.

Table 2: Obtained results with two different methods

\begin{tabular}{|c|c|c|c|c|c|c|}
\hline & $\sum \mathrm{C}_{\mathrm{i}}$ & $\sum \mathrm{T}_{\mathrm{i}}$ & \multirow[b]{2}{*}{ Deviation } & \multirow{2}{*}{ ACS-VNS } & \multirow[b]{2}{*}{ Tabu Search TS } & \multirow{2}{*}{ Deviation } \\
\hline & ACS-VNS & Tabu Search TS & & & & \\
\hline Tai 20_10_1 & 20911 & 20911 & 0 & 4043 & 4450 & -407 \\
\hline Tai 20_10_2 & 22440 & 22559 & -119 & 5411 & 5775 & -364 \\
\hline Tai 20_10_3 & 19877 & 19915 & -38 & 3835 & 4144 & -309 \\
\hline Tai 20_10_4 & 18710 & 18710 & 0 & 3466 & 3729 & -263 \\
\hline Tai 20_10_5 & 18679 & 18713 & -34 & 2515 & 2730 & -215 \\
\hline Tai 20_10_6 & 19245 & 19245 & 0 & 3345 & 3854 & -509 \\
\hline Tai 20_10_7 & 18448 & 18481 & -33 & 3247 & 3687 & -440 \\
\hline Tai 20_10_8 & 20241 & 20254 & -13 & 4599 & 4811 & -212 \\
\hline Tai 20_10_9 & 20330 & 20353 & -23 & 4124 & 4145 & -21 \\
\hline Tai 20_10_10 & 21320 & 21323 & -3 & 4403 & 4704 & -301 \\
\hline
\end{tabular}

Based on the results illustrated in Table 2, we easily note the performance of ACS-VNS approach compared to the Tabu search approach (TS) proposed by Allouche (2010). $70 \%$ of results have a negative deviation versus $30 \%$ of nil deviation for the total flowtime criterion. For the total tardiness, $100 \%$ of results have a negative deviation. Two other parameters that perform ACS-VNS approach are the average of computation time and the average of obtaining satisfaction level of problems which are referred by Tables 3 and 4 respectively.

According to the results displayed in Table 3 , it is clear that our approach is more efficient than TS method. In a large cases of scheduling problem $(20$ tasks-5machines, 20 tasks-10 machines, 20 tasks-20 machines, 50 tasks- 5 machines) the average of 
computation time needed by ACS_VNS to display the compromise solution is very low compared to TS.

Table 3: comparison of average computation time

\begin{tabular}{ccc}
\hline & \multicolumn{2}{c}{ Computational Time (seconds) } \\
\hline Problem Size & ACS-VNS & TS \\
$20 \_5$ & 0.5389 & 0.79 \\
$20 \_10$ & 2.2655 & 11.528 \\
$20 \_20$ & 5.5369 & 25.229 \\
$50-5$ & 12.5296 & 19.087 \\
$50 \_10$ & 3171.2 & 47.301 \\
\hline
\end{tabular}

Indeed, for scheduling problem characterized by 50 tasks and 10 machines, the average of computational time is lower than presented by ACOVNS. This can be explained by the fact that the performance of our approach tends to deteriorate when the problem size increases.

Table 4: comparison of average satisfaction level

\begin{tabular}{ccc}
\hline & \multicolumn{2}{c}{ Satisfaction Level } \\
\hline Problem Size & ACS-VNS & TS \\
$20 \_5$ & 0.99 & 1 \\
$20 \_10$ & 0.98 & 0.95 \\
$20 \_20$ & 0.92 & 0.91 \\
$50-5$ & 1 & 1 \\
$50 \_10$ & 0.96 & 0.95 \\
\hline
\end{tabular}

On the other hand, the average of the satisfaction level of the decision maker's for most of the problems are closed to 1 which mean that the decision maker is fully satisfied and the obtained sequences meet his preferences and aspiration levels.

\section{Conclusion}

In this study, we have presented a new hybrid metaheuristic approach to solve permutation flowshop scheduling problems which incorporates explicitly the decision maker's preferences. The proposed approach based on Ant colony algorithm and the variable neighborhood search noted by ACS_VNS is compared to Tabu search method presented by Allouche (2010). Indeed, it has performed competitively with the best results obtained by Allouche (2010).

The numerical experiments were done on a different set of problems with different sizes. The quality of the obtained solutions confirms the efficiency of the ACS_VNS compared to the TS. Using ACS_VNS, we can produce much better results than TS. It can be used for dependent as well as independent criteria. The obtained solutions minimize the deviation between the achievement level of each scheduling objective and its ideal points.

On the other hand, the proposed approach ACS_VNS is flexible, friendly use, it can support more than three criteria and the decision makers can provide easily the required information for establishing the satisfaction functions. Finally, it can be considered as a good tool for multicriteria scheduling problem, especially since it does not necessitate a long computational time.

\section{Acknowledgment}

This paper has been supported by Deanship of Scientific Research of the Northern Border University under the reference number 6-14-1436-5

\section{References}

Allouche M, Aouni B, Martel J, Loukil T, and Rebaï A (2009). Solving multi-criteria scheduling flow shop problem through compromise programming and satisfaction functions. European Journal of Operational Research, 192(2): 460-467.

Allouche MA (2010). Manager's preferences modeling within multi-criteria flowshop scheduling problem: A metaheuristic approach. International Journal of Business Research and Management ISSN, 1(2): 33-45.

Chang P, Chen S, Fan C, and Chan C (2008). Genetic algorithm integrated with artificial chromosomes for multi-objective flowshop scheduling problems. Applied Mathematics and Computation, 205(2): 550-561.

Daniels RL and Chambers RJ (1990). Multiobjective flow-shop scheduling. Naval Research Logistics (NRL), 37(6): 981-995.

Dorigo M, Maniezzo V, and Colorni A (1996). Ant system: optimization by a colony of cooperating agents. IEEE Transactions on Systems, Man, and Cybernetics, Part B (Cybernetics), 26(1): 29-41.

Eren $\mathrm{T}$ (2007). A multicriteria flowshop scheduling problem with setup times. Journal of Materials Processing Technology, 186(1): 60-65.

Gagné C, Gravel M, and Price W (2004). Optimisation multiobjectifs à l'aide d'un algorithme de colonie de fourmis. INFOR: Information Systems and Operational Research, 42(1): 23-42.

Gagné C, Gravel M, and Price W (2005). Using metaheuristic compromise programming for the solution of multipleobjective scheduling problems. Journal of the Operational Research Society, 56(6): 687-698.

Gambardella LM and Dorigo M (1995). Ant-Q: A reinforcement learning approach to the traveling salesman problem. In the $12^{\text {th }}$ International Conference on Machine Learning, Morgan Kaufmann, Burlington, USA: 252-260.

Gambardella LM and Dorigo M (1996). Solving symmetric and asymmetric TSPs by ant colonies. In the IEEE International Conference on Evolutionary Computation, IEEE, Nagoya, Japan: 622-627. https://doi.org/10.1109/ICEC.1996.542672

Gangadharan R and Rajendran C (1994). A simulated annealing heuristic for scheduling in a flowshop with bicriteria. Computers and Industrial Engineering, 27(1-4): 473-476.

Hansen $\mathrm{P}$ and Mladenović N (1997). Variable neighborhood search for the p-median. Location Science, 5(4): 207-226.

Javadi B, Saidi-Mehrabad M, Haji A, Mahdavi I, Jolai F, and Mahdavi-Amiri N (2008). No-wait flow shop scheduling using fuzzy multi-objective linear programming. Journal of the Franklin Institute, 345(5): 452-467.

Lin B, Lu C, Shyu S, and Tsai C (2008). Development of new features of ant colony optimization for flowshop scheduling. International Journal of Production Economics, 112(2): 742755.

Loukil T, Teghem J, and Tuyttens D (2005). Solving multi-objective production scheduling problems using metaheuristics. European Journal of Operational Research, 161(1): 42-61.

Martel J and Aouni B (1990). Incorporating the decision-maker's preferences in the goal-programming model. Journal of the Operational Research Society, 41(12): 1121-1132.

Qian B, Wang L, Huang D, Wang W, and Wang X (2009). An effective hybrid DE-based algorithm for multi-objective flow 
shop scheduling with limited buffers. Computers and Operations Research, 36(1): 209-233.

Rajendran C and Ziegler H (2004). Ant-colony algorithms for permutation flowshop scheduling to minimize makespan/total flowtime of jobs. European Journal of Operational Research, 155(2): 426-438.

Ruiz R and Allahverdi A (2009). Minimizing the bicriteria of makespan and maximum tardiness with an upper bound on maximum tardiness. Computers and Operations Research, 36(4): 1268-1283.

Taillard E (1993). Benchmarks for basic scheduling problems. European Journal of Operational Research, 64(2): 278-285.

Tavakkoli-Moghaddam R, Rahimi-Vahed AR, and Mirzaei AH (2007). Solving a bi-criteria permutation flow shop problem using immune algorithm. In the IEEE Conference on Computational Intelligence in Scheduling, IEEE, Honolulu, USA: 49-56. https://doi.org/10.1109/SCIS.2007.367669

T'kindt V and Billaut JC (2002). Multicriteria Scheduling: Theory, Models and algorithms. Springer-Verlag, Berlin, Germany.

Varadharajan $\mathrm{T}$ and Rajendran C (2005). A multi-objective simulated-annealing algorithm for scheduling in flowshops to minimize the makespan and total flowtime of jobs. European Journal of Operational Research, 167(3): 772-795.

Zeleny M (1973). Compromise programming. In: Zeleny $M$ and Cochrane JL (Eds.), Multiple criteria decision making: 262301. University of South Carolina Press, South Carolina, Columbia. 